\title{
AUC for SPECT: Is gender bias inappropriate
}

\author{
Rupa Mehta, $M D,{ }^{a}$ and Kim Allan Williams, $M D^{b}$
}

\section{See related article, pp. 588-594}

The appropriate use criteria (AUC) were created to guide physicians on ordering practices in various clinical scenarios. ${ }^{1}$ Most cardiologists are now somewhat familiar with the AUC but have not yet incorporated them into their clinical practice. The American College of Cardiology (ACC) has created the FOCUS Campaign to increase use of the AUC with a commitment to reduce the incidence of inappropriate imaging in the next few years. In addition, lab accreditation will now require an evaluation of appropriateness, further stressing the importance of incorporating the AUC into the clinical setting.

It has already been demonstrated that there are numerous challenges to incorporating the AUC into the clinical arena including: (1) obtaining all of the necessary information to categorize patients, whether this is done at point of ordering or point of service, (2) definitions of angina vary amongst practitioners, and (3) training lab personnel to use the AUC and to categorize patients properly. ${ }^{2}$ As clinicians, we can appreciate that many patients do not easily fit into a particular category and that clinical judgment plays a significant role in when and why we order a particular test. There are subsets of patients that have already been shown to have high rates of inappropriate testing. This includes asymptomatic patients and women. In fact, the most common inappropriate indications are in asymptomatic patients. $^{2}$ To further guide clinical decision making in this subset of patients, the American Society of Nuclear Cardiology has recently published an information

From the Section of Cardiology, Department of Medicine, ${ }^{a}$ University of Chicago Medical Center, Chicago, IL; Division of Cardiology, ${ }^{b}$ Wayne State University School of Medicine, Detroit, MI, USA.

Reprint requests: Rupa Mehta, MD, Section of Cardiology, Department of Medicine, University of Chicago Medical Center, 5841 S. Maryland, MC 2090, Chicago, IL 60638; rmehta@medicine. bsd.uchicago.edu.

J Nucl Cardiol 2011;18:538-40.

1071-3581/\$34.00

Copyright (C) 2011 American Society of Nuclear Cardiology.

doi:10.1007/s12350-011-9374-z statement on the use of single perfusion computed tomography myocardial perfusion imaging (SPECT MPI) in asymptomatic patients. ${ }^{3}$ There is, however, a paucity of data regarding the AUC in women.

In this issue of the Journal of Nuclear Cardiology, Gupta et $\mathrm{al}^{4}$ investigated gender disparity in the use of the appropriateness criteria. They had several notable findings. They found that inappropriate studies were more likely to be ordered in women than in men $(68 \%$ vs $32 \%)$. The average age of women in the inappropriate group was $48 \pm 9$ years. Uncertain studies were also more likely to be ordered in women than in men $(82 \%$ vs $18 \%$ ) with an average age of women in this group of $47 \pm 6$ years. The average age of the total cohort was $62 \pm 14$ years. Younger patients were more likely to have inappropriate or uncertain studies as would be expected with a lower-risk patient. It would have been informative if the study had indicated what portion of these inappropriate studies was normal. They mention that 8 studies in the inappropriate and uncertain groups had high-risk test results but do not comment on the degree of abnormality. Their data supports previously published data by Mehta et $\mathrm{al}^{5}$ that women are more likely to have an inappropriate study ( $74 \%$ vs $26 \%$ ). Mehta et al also showed that normal studies are more frequent in women than in men that were ordered for inappropriate indications. However, they also noted that a quarter of women in the inappropriate group and almost half of the women in the uncertain group had SPECT abnormalities. When abnormal, women in the inappropriate and appropriate groups had lower summed stress scores (smaller defects) than men. Although these results show lower summed stress scores, the presence of an abnormal result could potentially alter medical management in a patient with no prior history of CAD. Downstream changes could include the addition of aspirin, a statin or more aggressive risk factor reduction through lifestyle modifications.

Gupta et al report that women undergoing SPECT MPI have a relative risk of 2.69 that the test will be classified as inappropriate and a relative risk of 6.0 that the test will be classified as uncertain with all other factors being equal. Hendel et $\mathrm{al}^{2}$ have also shown in a multi-center study that female sex is an independent risk factor for receiving an inappropriate classification, increasing the likelihood of being considered inappropriate by 2.5 times for women compared to men even 
when controlling for risk factors and symptoms. These two studies show a similar relative risk. In the Hendel study, the best predictor of an inappropriate classification was asymptomatic status of the patient (odds ratio $=22.5$ ). The current study does not comment on other predictors of risk and focuses only on the impact of gender on risk.

It is interesting to note that $96 \%$ of the tests in this study were ordered for chest pain. Only five women were asymptomatic of which two were excluded due to missing data and the inability to categorize the patient. When considering symptoms in this patient population, it is notable that women often present with atypical symptoms and do not fit the classic Diamond and Forrester definition of angina which includes: (a) the presence of substernal chest pain, (b) which occurs with exertion, and (c) is relieved by rest or nitroglycerin. ${ }^{1,6,7}$ Women are more likely to present with shortness of breath, fatigue, or atypical chest pain. Per the 2009 AUC, ${ }^{1}$ dyspnea is considered an anginal equivalent, but it is unclear if this should be categorized as atypical chest pain or non-anginal chest pain. This distinction could dramatically alter the appropriateness category for a patient in a given clinical scenario. The authors do suggest that a different tool for pre-test risk assessment of the symptomatic patient may allow for more accurate assessment of women but do not discuss a specific tool.

On gender reversal analysis, it was noted that 25 studies $(8 \%)$ would go from appropriate to inappropriate, and that 21 studies $(7 \%$ ) would go from inappropriate to appropriate. Since a large proportion of their inappropriate group were women and of a younger age (average age 48), it is not surprising that there is less correlation during gender reversal. The AUC is clearly not gender independent. Most of the patients in this trial were symptomatic and in the AUC, as previously mentioned, symptomatic women are categorized according to Diamond and Forrester Criteria. This criteria classifies all women $<50$ years of age as being low risk if they have non-anginal or atypical angina, regardless of risk factors and co-morbidities. It is well known that most women present with atypical chest pain and, accordingly, would be classified as low risk in this younger patient cohort.

Lastly, Gupta et al report on the type of ordering physician. They found that primary care physicians were more likely to order studies overall but were less likely to order appropriate studies $(79 \%$ PCP vs $92 \%$ cardiologists). Of the inappropriate and uncertain studies, the vast majority were ordered by primary care physicians (74\% of inappropriates, 94\% of uncertains). Specifically, looking at women, cardiologists were more likely to order tests classified as appropriate (86\% vs $71 \%$ ). In both ordering groups, however, men had more appropriate studies than women. With the higher rate of inappropriate testing in women, it is clear that physicians have a lower "threshold" to order a stress test in women even when it does not fall into an appropriate ordering classification. This may reflect an understanding and appreciation for the atypical presentation of female cardiac patients.

The data provided by Gupta et al highlights the importance of critically reviewing ordering practices by gender. There are, however, limitations to the current study. It does not comment on the results or outcomes of the inappropriate and uncertain studies as the total number of patients in these two subgroups is quite small. It would be prudent to document what proportion of "inappropriate" studies are actually abnormal and may change patient management in order to prove the validity of some inappropriate testing. We particularly need to evaluate the "number needed to image", in order to find a clinically important stress test result in the inappropriate group, which could help define how such patients should be managed. The importance of clinical judgment in patients that present with atypical chest pain must be preserved. In an era where reimbursement is being challenged for patients that do not fall into specific criteria, we, as practitioners must fight to maintain our autonomy when ordering a test that might be deemed "inappropriate", when based on clinical judgment. We must provide evidence that certain patient cohorts, such as women, may require a higher "inappropriate", imaging rate to diagnose disease, and that the use of these tests does indeed change long-term management and potentially save lives.

\section{References}

1. Hendel RC, Berman DS, Di Carli MF, Heidenreich PA, Henkin RE, Pellikka PA, et al. ACCF/ASNC/ACR/AHA/ASE/SCCT/SCMR/ SNM 2009 appropriate use criteria for cardiac radionuclide imaging: a report of the American College of Cardiology Foundation Appropriate Use Criteria Task Force. J Am Coll Cardiol 2009;53:2201-29.

2. Hendel RC, Cerqueira M, Douglas PS, Caruth KC, Allen JM, Jensen NC, et al. A multicenter assessment of the use of singlephoton emission computed tomography myocardial perfusion imaging with appropriateness criteria. J Am Coll Cardiol 2010;55:156-62.

3. Hendel RC, Abbott BG, Bateman TM, Blankstein R, Calnon DA, Leppo JA, et al. The role of radionuclide myocardial perfusion imaging for asymptomatic individuals. J Nucl Cardiol 2011;18:3-15.

4. Gupta A, Tsiaras SV, Dunsiger SI, Tilkemeier PL. Gender disparity and the appropriateness of myocardial perfusion imaging. J Nucl Cardiol 2011;18. doi:10.1007/s12350-011-9368-X.

5. Mehta R, Ward RP, Chandra S, Agarwal R, Williams KA. Evaluation of the American College of Cardiology Foundation/ American Society of Nuclear Cardiology appropriateness criteria for SPECT myocardial perfusion imaging. J Nucl Cardiol 2008; 15:337-44. 
6. Diamond GA, Forrester JS. Analysis of probability as an aid in the clinical diagnosis of coronary-artery disease. $N$ Engl J Med 1979;300:1350-8.

7. Gibbons RJ, Balady GJ, Bricker T, Chaitman BR, Fletcher GF, Froelicher VF, et al. ACC/AHA 2002 guideline update for the management of patients with chronic stable angina, summary article: a report of the American College of Cardiology/American Heart Association Task Force on Practice Guidelines (Writing Committee on the Management of Patients with Chronic Stable Angina). J Am Coll Cardiol 2003;41:159-68. 